\title{
Spontaneous, synchronized, and corrective timing behavior in cerebellar lesion patients
}

Citation for published version (APA):

Schwartze, M., Keller, P. E., \& Kotz, S. A. (2016). Spontaneous, synchronized, and corrective timing behavior in cerebellar lesion patients. Behavioural Brain Research, 312, 285-293.

https://doi.org/10.1016/j.bbr.2016.06.040

Document status and date:

Published: 01/10/2016

DOI:

10.1016/j.bbr.2016.06.040

Document Version:

Publisher's PDF, also known as Version of record

Document license:

Taverne

Please check the document version of this publication:

- A submitted manuscript is the version of the article upon submission and before peer-review. There can be important differences between the submitted version and the official published version of record.

People interested in the research are advised to contact the author for the final version of the publication, or visit the DOI to the publisher's website.

- The final author version and the galley proof are versions of the publication after peer review.

- The final published version features the final layout of the paper including the volume, issue and page numbers.

Link to publication

\footnotetext{
General rights rights.

- You may freely distribute the URL identifying the publication in the public portal. please follow below link for the End User Agreement:

www.umlib.nl/taverne-license

Take down policy

If you believe that this document breaches copyright please contact us at:

repository@maastrichtuniversity.nl

providing details and we will investigate your claim.
}

Copyright and moral rights for the publications made accessible in the public portal are retained by the authors and/or other copyright owners and it is a condition of accessing publications that users recognise and abide by the legal requirements associated with these

- Users may download and print one copy of any publication from the public portal for the purpose of private study or research.

- You may not further distribute the material or use it for any profit-making activity or commercial gain

If the publication is distributed under the terms of Article $25 \mathrm{fa}$ of the Dutch Copyright Act, indicated by the "Taverne" license above, 
Research report

\title{
Spontaneous, synchronized, and corrective timing behavior in cerebellar lesion patients
}

\author{
Michael Schwartze ${ }^{\mathrm{a}, \mathrm{b}, *}$, Peter E. Keller ${ }^{\mathrm{c}}$, Sonja A. Kotz ${ }^{\mathrm{a}, \mathrm{b}}$ \\ a Maastricht University, Universiteitssingel 40, 6229 Maastricht, The Netherlands \\ ${ }^{\mathrm{b}}$ Max Planck Institute for Human Cognitive and Brain Sciences, Stephanstrasse 1A, 04103 Leipzig, Germany \\ c The MARCS Institute, University of Western Sydney, Penrith NSW 2751, Sydney, Australia
}

\section{H I G H L I G H T S}

- Cerebellar patients and controls performed self-paced and paced sensorimotor tasks.

- The results confirm a temporal processing dysfunction in cerebellar lesion patients.

- Lesions increase asynchronies, decrease tempo sensitivity, impair error correction.

- The results are compatible with a sensory predictive account of cerebellar function.

\section{A R T I C L E I N F O}

\section{Article history:}

Received 14 April 2016

Received in revised form 16 June 2016

Accepted 20 June 2016

Available online 21 June 2016

\section{Keywords:}

Brain injury

Sensorimotor synchronization

Adaptation

Temporal processing

Prediction

\begin{abstract}
A B S T R A C T
To successfully navigate through and interact with a dynamic environment it is necessary to acquire and use adequate temporal information to guide behavior. Apart from several areas in the cerebral cortex and cortico-striatal networks, the cerebellum has been proposed to engage in the processing of temporal information. Damage to the cerebellum can impair precise event-based temporal processing in motor and non-motor behavior. To further substantiate cerebellar contributions to temporal processing and to explore its specific role in adapting to a dynamic environment, we investigated sensorimotor temporal processing in ten patients with cerebellar lesions and a corresponding number of healthy matched controls. Experimental tasks included simple self-paced repetitive finger-tapping (spontaneous motor tempo), temporally non-adaptive (isochronous pacing) and adaptive (tempo-changing pacing) sensorimotor synchronization with auditory sequences (synchronization-continuation tapping), and a perceptual tempo judgment. The results indicate that patients' performance diverges systematically from controls on several measures. Cerebellar patients demonstrate more variable self-paced tapping, larger negative asynchronies when synchronizing with isochronous pacing sequences, altered automatic error correction responses to tempo changes (phase correction), and decreased perceptual sensitivity to these perturbations, especially for small accelerations. These findings confirm imprecise temporal processing in cerebellar patients, and hint at a specific impairment in the tens-of-milliseconds range preceding critical events, in line with a temporally predictive account of cerebellar function. Moreover, this cerebellar profile complements previous findings concerning dysfunctional temporal processing in basal ganglia patients assessed with the same experimental setup, suggesting structural and functional differentiation within an integrative temporal processing network.
\end{abstract}

(c) 2016 Elsevier B.V. All rights reserved.
* Corresponding author at: Maastricht University, Department of Neuropsychology and Psychopharmacology, Universiteitssingel 40, 6229 Maastricht, The Netherlands.

E-mail address: michael.schwartze@maastrichtuniversity.nl (M. Schwartze).

\section{Introduction}

Reciprocal polysynaptic connections between the cerebellum, thalamus, cerebral cortex, and subcortical areas establish the structural basis for functional interactions, and an active role of the cerebellum in motor and non-motor behavior [1-6]. A common denominator in cerebellar function across these domains is temporal processing, conceived as the ability to encode, decode, and evaluate the temporal structure of sensory and sensorimotor 
events. In addition to the basal ganglia, prefrontal-, and supplementary motor areas, the cerebellum is among the brain regions consistently associated with temporal processing [7-11]. More specifically, the cerebellar timing hypothesis suggests that the cerebellum generates a precise internal representation of the temporal relation between salient events in the subsecond range [12-14].

Another denominator in cerebellar function is a conglomerate of related functions, namely the interplay of preparation, prediction, and attention, conceived as a dynamic process of constant adaptation to internal and external variation [15]. Cerebellar circuitry involved in predicting sensory consequences of action ("forward-modelling" [16]) has been suggested to generate a temporal signal, which, potentially in concert with pre-supplementary motor cortices, guides sensory prediction and the temporal orienting of attention [17]. This mechanism is reminiscent of a more general concept of attention and the allocation of attention in time as formulated in the framework of dynamic attending theory [18]. This theory considers naturally occurring internal variations (fluctuations) of attention directly sensitive to the temporal structure of external stimulation. Modelled as a complex of adaptive oscillations (attending rhythms), these fluctuations may establish a stimulus-driven, synchronized attending mode, which allows prediction of the temporal locus of future events. From this perspective, the temporal structure of events determines efficient allocation of attention and thereby the quality of integrated percepts [18].

The question arises whether the precise representation of event timing, forward-modelling, and the efficient allocation of attention converge into a generalizable function in behavior that requires or at least benefits from real-time prediction $[19,20]$. This assumption is essentially a variant of the idea that the cerebellum contributes to precise sensory discrimination and integration, and controls the acquisition of sensory data to increase the efficiency of processing in cerebral cortex [21]. These cerebellar contributions to temporal aspects of behavior are perhaps most evident (but not limited to) tasks, in which sensory processing concurs in conjunction with movement $[22,23]$. Notwithstanding functional specialization within specific cerebellar subregions, the cerebellum as a whole may thus instantiate a sensorimotor structure, which modulates activity in cerebral cortex to optimize behavior in terms of temporal precision and coherence, synchronization, and temporal prediction as facets of ongoing interactions with a dynamic environment. On this account, damage to the cerebellum should reduce the efficiency of these operations, leading to suboptimal temporal precision, which may cascade into a broad range of motor and non-motor behaviors.

In the current study, we compared the performance of patients with cerebellar lesions and healthy controls in a set of sensorimotor tasks that require a relatively high degree of temporal precision. Self-paced generation of temporal regularity (spontaneous motor tempo, SMT), paced synchronization and unpaced reproduction, as well as adaptation and perceptual sensitivity to tempo changes were assessed using previously established adaptive finger-tapping paradigms in combination with a modelling approach that has been shown to differentiate between automatic and attention-dependent forms of error correction in adaptive sensorimotor synchronization [24,25]. Firstly, we expected patients' performance to diverge systematically from controls in all domains tested: spontaneous, paced, and perceptual behaviour, and to exhibit a profile of dysfunctions that would be compatible with the proposed role of the cerebellum in precise subsecond temporal processing in production and perception as suggested by the cerebellar timing hypothesis. Secondly, based on the current modelling approach we also expected this profile to reflect dysfunctional automatic mechanisms, thus differing from the previously obtained profile of basal ganglia patients, who demonstrated dysfunctional attention-dependent error correction in adaptive sensorimotor synchronization [25]. The emergence of such a pattern would substantiate the notion of cerebellum and basal ganglia as nodes within an integrative temporal processing network, and specify this global function on the basis of a differential engagement of attention.

\section{Material and methods}

\subsection{Participants}

10 chronic-stage right-handed patients with cerebellar lesions (mean age: 45.0, SD: 14.0, range: 25-70 years; 3 women) and 10 healthy matched controls (mean age: 46.8 , SD: 13.1 years) participated in the experiment (Table 1, Fig. 1). All participants provided informed written consent with the study. Individual controlpatient pairings were matched in terms of handedness, gender, age $(+/-2$ years), and education (in years). None of the participants had professional musical expertise. Both groups were recruited via databases at the Max Planck Institute for Human Cognitive and Brain Sciences in Leipzig, Germany. None of the participants had prior experience with finger-tapping in an experimental setup. The study was conducted in accordance with the World Medical Association Declaration of Helsinki and was approved by the ethics committee of the University of Leipzig.

\subsection{Structural magnetic resonance imaging}

High-resolution T1-weighted magnetic resonance scans were obtained for each patient on Bruker BioSpin (BioSpin GmbH, Rheinstetten, Germany) or Siemens TrioTim (Siemens Healthcare, Erlangen, Germany) magnetic resonance systems at $3 \mathrm{~T}$ using a 32channel phased-array head array coil and an MP-RAGE sequence [26]. The resulting images were segmented and spatially normalized to Montreal Neurological Institute (MNI) space by means of a unified segmentation approach [27] in SPM (SPM8, Wellcome Department of Imaging Neuroscience, London, http://www.fil.ion.

Table 1

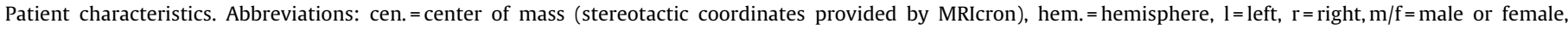
vol. = lesion volume in cc.

\begin{tabular}{|c|c|c|c|c|c|c|}
\hline no. & $\mathrm{m} / \mathrm{f}$ & age & vol. & cen. $(x, y, z)$ & hem. & aetiology \\
\hline 1 & $\mathrm{~m}$ & 25 & 48.0 & $77,38,35$ & $1, \mathrm{r}$ & arteriovenous malformation, intracranial haemorrhage \\
\hline 2 & $\mathrm{~m}$ & 30 & 30.1 & $52,53,46$ & 1 & superior cerebellar artery aneurysm, intracranial haemorrhage \\
\hline 3 & $\mathrm{~m}$ & 45 & 4.9 & $84,64,47$ & $1, \mathrm{r}$ & basilar artery aneurysm, superior cerebellar artery infarction \\
\hline 4 & $\mathrm{~m}$ & 70 & 1.6 & $63,31,35$ & 1 & posterior inferior cerebellar artery infarction \\
\hline 5 & $\mathrm{~m}$ & 59 & 7.1 & $89,37,26$ & $\mathrm{r}$ & posterior inferior cerebellar artery infarction \\
\hline 6 & $\mathrm{f}$ & 38 & 0.3 & $121,48,27$ & $\mathrm{r}$ & posterior inferior cerebellar artery infarction \\
\hline 7 & $\mathrm{f}$ & 45 & 7.9 & $62,41,24$ & $1, \mathrm{r}$ & posterior inferior cerebellar artery infarction \\
\hline 8 & $\mathrm{~m}$ & 55 & 14.5 & $57,40,24$ & 1 & posterior inferior cerebellar artery infarction \\
\hline 9 & $\mathrm{f}$ & 33 & 8.8 & $84,49,37$ & $\mathrm{r}$ & medulloblastoma resection \\
\hline 10 & $\mathrm{~m}$ & 50 & 2.4 & $88,52,15$ & $\mathrm{r}$ & posterior inferior cerebellar artery infarction \\
\hline
\end{tabular}




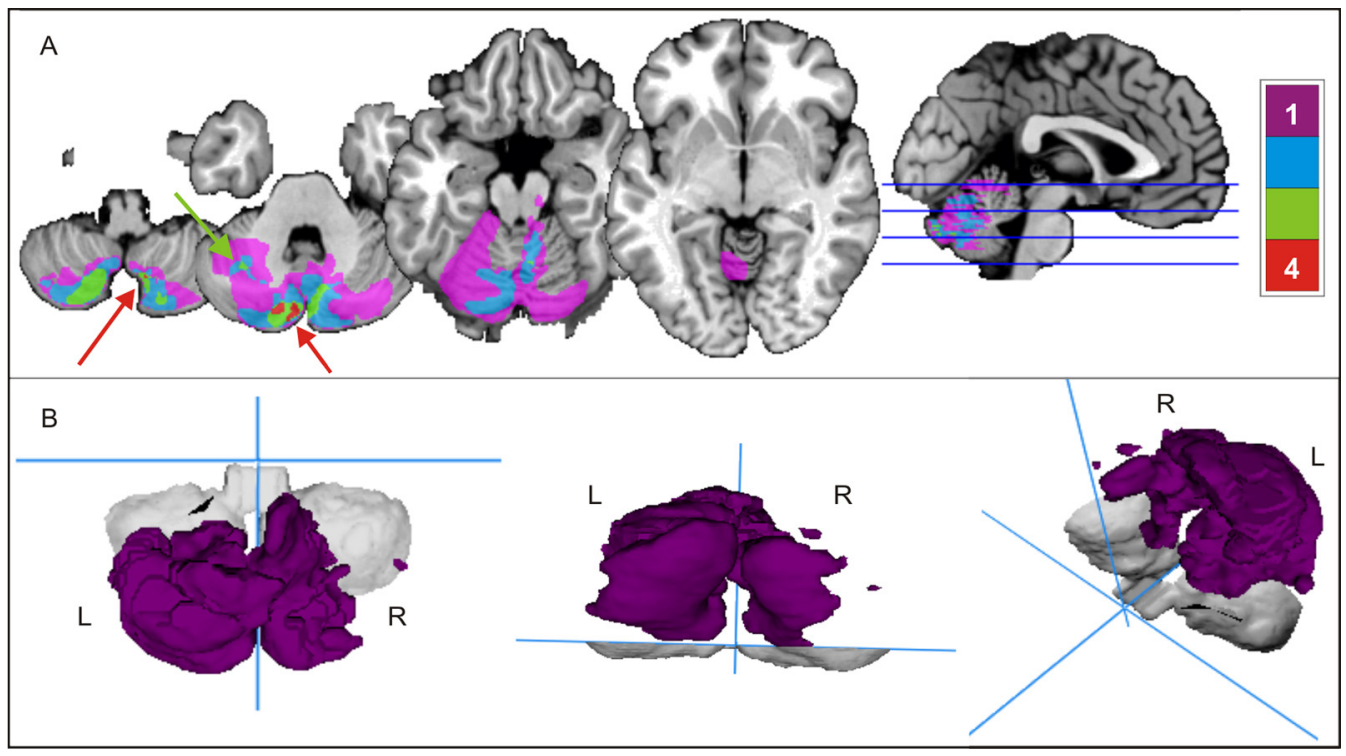

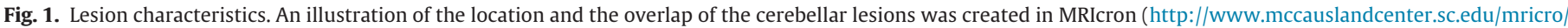

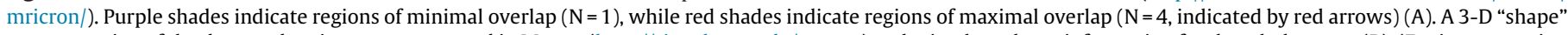

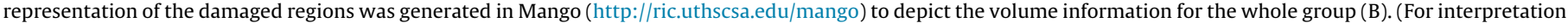
of the references to color in this figure legend, the reader is referred to the web version of this article.)

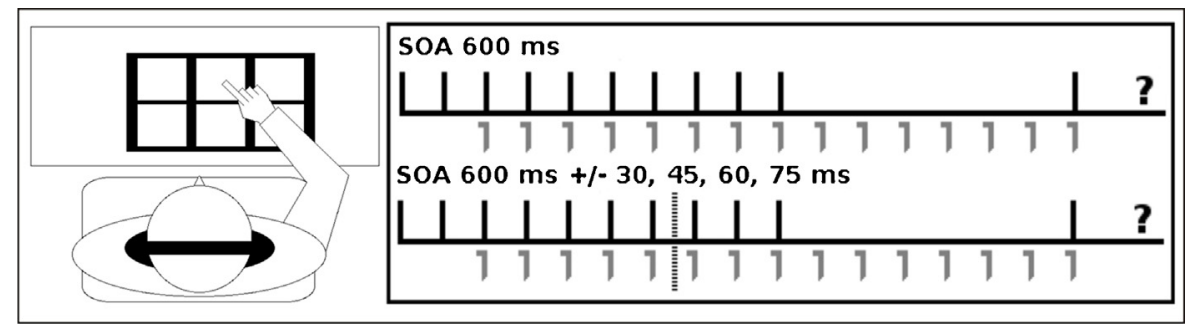

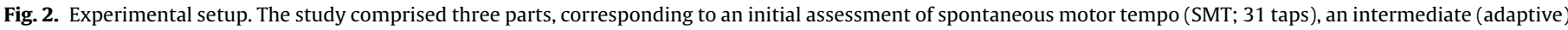

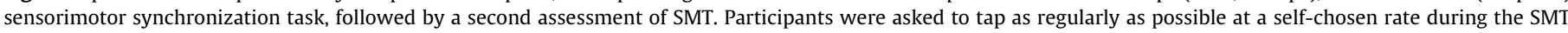

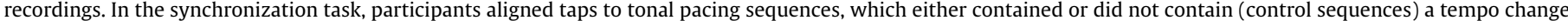

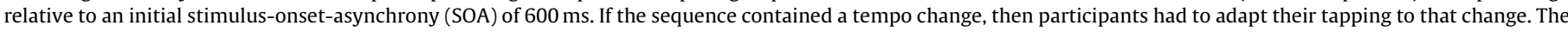

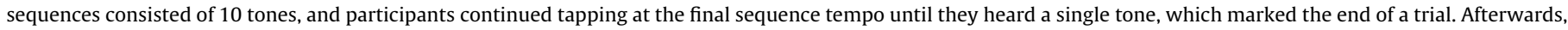
they reported whether they had perceived the sequence as getting faster, slower, or as maintaining a constant tempo.

ucl.ac.uk/spm). Volumes of interest corresponding to individual lesions were delineated manually on axial slices of the normalized images in MRIcron [28] and plotted onto a scalp-stripped T1-weighted single subject template (Colin27_T1_seg_MNI.nii, available via http://brainmap.org/ale/index.html; Fig. 1). Finally, the total volume $\left(\mathrm{cm}^{3}\right)$ and the center of mass (cartesian coordinates) of each lesion as provided by MRIcron were extracted for subsequent use in correlational analyses, providing information about lesion size and a form of approximation of lesion location in terms of the anterior-posterior (x), lateral (y), and superior-inferior (z) dimensions of the center of mass.

\subsection{Data collection and analysis}

The current protocol was the same as in previous work ([25]; Fig. 2). Stimulus presentation and data recording was controlled by custom software written in MAX (cycling74.com) running on a PC. The SMT of each participant was assessed before (SMT1) and after (SMT2) the adaptive synchronization task. Participants sat on a chair and tapped for 31 taps (30 inter-tap-intervals, ITIs) on the rubber surface of an electronic percussion pad (Roland SPD-6) connected to the Musical Instrument Digital Interface (MIDI) port of the PC. They were asked to tap as regularly as possible at a self-chosen rate until they heard a single tone (E7) presented via headphones
(Sennheiser HD 202). All participants were allowed to familiarize themselves with the setup and to test different styles of tapping on different locations on the pad, but the experimenter did not demonstrate the task to avoid potential influences on individual performance. All participants elected to tap with the index finger of their right hand and to rest their hand on the pad. For SMT, variables of interest were tapping rate (mean inter-tap-interval (M_ITI)) and tapping variability (coefficient of variation (CV_ITI), obtained by dividing the ITI standard deviation by M_ITI).

The same technical setup was used during the adaptive synchronization task. In this case, participants were asked to synchronize their taps with an auditory pacing sequence ( $600 \mathrm{~ms}$ stimulusonset asynchrony) consisting of 10 piano tones $(\mathrm{C} 8,4176 \mathrm{~Hz})$, to adapt to any tempo change that might occur, and to continue tapping in the last perceived tempo after the end of the sequence until they heard a single lower pitch tone (E7), which signalled the end of the trial. All participants completed 100 pseudo-randomized trials in 10 blocks distributed over 9 conditions. Each block consisted of eight sequences that contained a tempo change relative to the $600 \mathrm{~ms}$ base tempo $(+/-30,45,60$, and $75 \mathrm{~ms})$ and two sequences without a tempo change that served as control sequences. All tempo changes occurred between the 7 th and the 8 th tone of the sequence. The order of the sequences was counterbalanced across the blocks. Participants were instructed to start tapping with the third tone of 

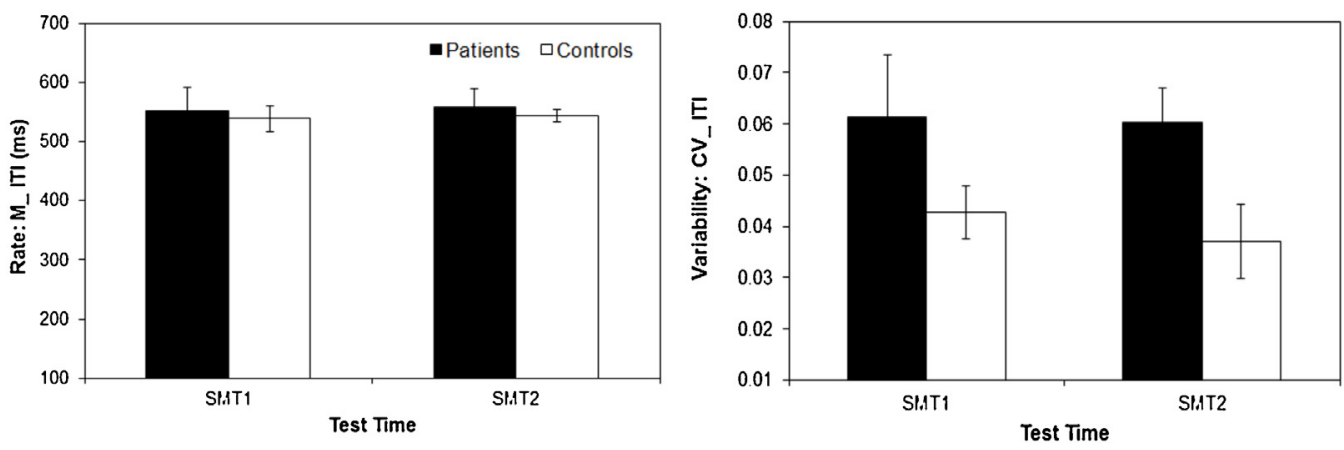

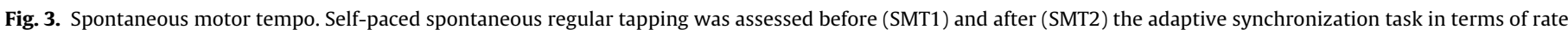

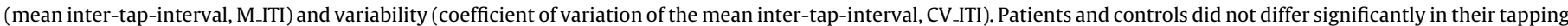
rates but patients displayed significantly increased tapping variability in comparison to controls in both assessments.

the pacing sequence. After they stopped tapping, participants indicated whether they had perceived an acceleration, deceleration, or no tempo change. An additional block of 10 trials served as training. There was a short break of approximately 5 min after block 5 .

Missing taps, e.g., due to insufficient tapping force, and misplaced taps (defined as lying outside a $-150 \mathrm{~ms}$ to $+130 \mathrm{~ms}$ time-window relative to the expected target position) were excluded from all further analyses. For sequences without a tempo change, variables of interest were the mean asynchrony between the tones and the taps (M_ASYN) and the corresponding variability (CV_ASYN), as well as M_ITI, and CV_ITI, calculated separately for synchronization and continuation tapping. The ability to adapt the tapping to an actual tempo change was assessed by means of several additional variables, i.e., adaptation indices computed separately for accelerations and decelerations, as well as phase- and period-correction estimates computed on the basis of five intervals (positions) of interest ( $\mathrm{s} 0, \mathrm{~s} 1, \mathrm{~s} 2, \mathrm{~s} 3, \mathrm{c})$. Position s0 corresponds to the interval terminated by the tap that coincided approximately with the first tone at the new sequence tempo, immediately followed by positions s1, s2, and s3. Thus, s3 corresponds to the interval initiated by the tap that coincided approximately with the last tone of the pacing sequence. Position $\mathrm{c}$ reflects the average interval produced during continuation tapping. These parameters were then submitted to a MATLAB (The Mathworks) implementation of the two-way error-corrective mechanism as originally proposed in a model by Mates ([29]; for a detailed description of the mathematical procedures applied for fitting data to this model see Ref. [24]) in order to obtain estimates for phase correction (compensation for gaps between the central availability of internal stimulus representations and response feedback) and period correction (compensation for discrepancies between an internal reference interval and an actual inter-stimulus-onset interval). Finally, perceptual sensitivity to the tempo changes was assessed in terms of individual d' scores computed separately for the various step changes. All variables of interest were analysed by means of repeated-measures ANOVA with a primary focus on the betweensubject factor group (patients vs. controls).

\section{Results}

All patients and controls were able to complete the experimental tasks without apparent signs of fatigue or needing additional breaks.

\subsection{Spontaneous motor tempo}

The spontaneous tapping rate (Fig. 3) in the patient group was similar for SMT1 (M_ITI: 551 ms, SD: 124) and SMT2 (M_ITI: 557 ms, SD: 99) and comparable to the rates observed in the control group for SMT1 (M_ITI: 539 ms, SD: 44) and SMT2 (M_ITI: 544 ms, SD: 35). This was substantiated by significant correlations between SMT1 and SMT2 in patients $(r=0.632, p=0.050)$ and in controls $(r=0.785, p=0.007)$, while a $2 \times 2$ ANOVA with the between-subject factor group (patients vs. controls) and the within-subject factor test time (SMT1 vs. SMT2) on M_ITI revealed no significant differences. However, the same type of ANOVA conducted for CV_ITI yielded a significant main effect of group $(F(1,18)=5.037, p=0.038$, $\left.\eta_{p}{ }^{2}=0.219\right)$, indicating generally more variable performance in patients.

In order to further explore structure-function relations within the patient group, M_ITI and CV_ITI for SMT1 and SMT2 were correlated first with lesion size and then with the three anatomical dimensions reflected by the center of mass for individual lesions. To address the problem of multiple comparisons, the sequentially rejective Holm-Bonferroni adjustment method [30] was applied in these and similar correlation analyses reported in the following. As each experimental variable of interest was correlated with four lesion-related variables, this procedure resulted in adjusted alpha levels of 0.0125 for the most significant test, 0.017 and 0.025 for the following, and 0.05 for the least significant test. This type of analysis yielded a significant positive correlation between the anteriorposterior dimension and M_ITI for SMT1 $(r=0.772, p=0.009)$ but not for SMT2 $(r=0.172, p=0.635)$. Subsequent exclusion of one patient whose performance fell outside a range of two standard deviations of the mean for SMT2 $(311 \mathrm{~ms})$ confirmed a similarly strong correlation between M_ITI for SMT1 and SMT2 in patients $(r=0.822, p=0.007)$ as in controls and yielded significant correlations between the anterior-posterior dimension and M_ITI for SMT1 ( $r=0.923, p=0.000)$ as well as SMT2 $(r=0.884, p=0.002)$, while it did not change the pattern of results for the corresponding ANOVAs. For the whole patient group, there were positive correlations between lesion size and CV_ITI for SMT1 $(r=0.821, p=0.004)$ and SMT2 $(r=0.792, p=0.006)$, suggesting that more anterior centers of mass for the respective lesions are associated with slower tapping rates, while larger lesions are generally associated with more variable tapping.

\subsection{Synchronization: sequences without tempo changes}

Trials in which the tempo of the pacing sequence remained constant served as a control for trials that required adaptation of the tapping rate to a changing pacing tempo. Patients and controls seemed to differ in several of the measures (Table 2).

To compare the group performance for the control sequences, independent-samples $t$-tests were conducted for the mean and the $\mathrm{CV}$ of the asynchronies between taps and pacing tones. Patients produced substantially larger $(t(18)=-7.919, p=0.000, d=3.535)$ and also more variable $(t(18)=4.115, p=0.001, d=1.803)$ nega- 
Table 2

Control sequences. Tapping performance for sequences without a tempo change was assessed in terms of the mean asynchrony (M_ASYN) and the coefficient of variation (CV_ASYN) between the tones of the pacing sequence and the corresponding taps. In addition, mean inter-tap-intervals (M_ITI) and coefficients of variation (CV_ITI) were computed separately for the paced synchronization (Syn) and the unpaced continuation (Con) phase.

\begin{tabular}{lllllll}
\hline & M_ASYN & CV_ASYN & M_ITI Syn & M_ITI Con & CV_ITI Syn & CV_ITI Con \\
\hline Patients & $-91 \mathrm{~ms}$ & 0.065 & $595 \mathrm{~ms}$ & $585 \mathrm{~ms}$ & 0.053 & 0.072 \\
Controls & $-21 \mathrm{~ms}$ & 0.035 & $599 \mathrm{~ms}$ & $599 \mathrm{~ms}$ & 0.043 & 0.048 \\
\hline
\end{tabular}

tive mean asynchronies than controls. In the latter case Levene's test for equality of variances proved significant $(F(1,18)=11.524$, $p=0.003$ ), with equal variances not assumed changing the result to $(t(10.150)=4.115, p=0.002)$. On average, the observed difference in the mean asynchronies corresponds to an additional $70 \mathrm{~ms}$, by which the taps in the patient group preceded the pacing stimuli relative to the $21 \mathrm{~ms}$ observed for the control group. Two separate $2 \times 2$ ANOVAs with the between-subject factor group (patients vs. controls), and the within-subject factor phase (synchronization vs. continuation) were conducted for M_ITI and CV_ITI. The ANOVA on M_ITI did not identify any significant differences. However, for CV_ITI this procedure yielded significant effects of group $\left(F(1,18)=5.652, p=0.029, \eta_{p}^{2}=0.239\right)$ and phase $(F(1,18)=31.226$, $\left.p=0.000, \eta_{p}^{2}=0.634\right)$, as well as an interaction of these factors $\left(F(1,18)=11.177, p=0.004, \eta_{p}^{2}=0.383\right)$. Subsequent direct comparisons revealed a group difference during the continuation phase $(t(18)=2.985, p=0.008, d=1.372)$ but not during the synchronization phase, indicating increased variability for self-paced tapping in patients. Again, Levene's test for equality of variances was significant $(F(1,18)=11.271, p=0.004)$, with equal variances not assumed changing the result to $(t(10.164)=2.985, p=0.013)$.

There were significant positive correlations between lesion size and CV_ITI for the synchronization phase $(r=0.818, p=0.004)$ and the continuation phase ( $r=0.825, p=0.003)$, essentially replicating findings for SMT concerning the relation of larger lesion extent and high tapping variability.

\subsection{Synchronization: sequences with tempo changes}

Performance for trials containing a tempo acceleration or deceleration was assessed on the basis of adaptation indices calculated separately for each participant and for each position of interest and type of tempo change. To this end, M_ITIs were plotted as a function of final sequence tempo (Fig. 4).

The slope of a linear regression line fitted to these functions was then taken as a measure of the ability to adapt to the final sequence tempo and to maintain this tempo during the continuation phase [25]. Adaptation indices (Fig. 5) were then analyzed by means of a $2 \times 2 \times 4$ ANOVA with the between-subject factor group (patients vs. controls), and the within-subject factors tempo (faster vs. slower) and position (s1 vs. s2 vs. s3 vs. c). When applicable, Greenhouse-Geisser corrected values are reported here and for similar analyses described below. While there was only a tendency for a main effect of group $\left(F(1,18)=3.314, p=0.085, \eta_{p}{ }^{2}=0.155\right)$, there was a significant effect of position $(F(3,54)=7.802, p=0.000$, $\left.\eta_{p}{ }^{2}=0.302\right)$ and an interaction of group $x$ position $(F(3,54)=3.523$, $p=0.021, \eta_{p}^{2}=0.164$ ).

Subsequent analyses revealed a group difference for position $\mathrm{s} 1$ only $(t(18)=-3.126, p=0.006, d=1.409)$, i.e., the first interval for which a reaction to the tempo change occurring during the preceding interval (position s0) can be expected. This result indicates the lack of initial overcorrection in the patient group contrary to behavior typically observed in healthy participants [31,32]. This phenomenon has been linked to the perceptual sensitivity towards the tempo change ([24]; see also Section 3.4). Visual inspection of the data showed that the lack of overcorrection at the group level was primarily due to four patients with adaptation indices $<0.9$ (indicating atypical undercorrection), whereas such behavior was entirely absent in the control group (all adaptation indices $>1.1$ ). To further explore the potential anatomical correlates of these particular profiles, we looked for overlap in lesion locations in the respective patients. There was a single small area of overlap for three out of four overlays, located in the left cerebellar crus I (Fig. 1, green arrow).

Informed by the findings of the preceding ANOVA, correlation analyses with lesion size and location were performed on the basis of averaged adaptation indices for each position of interest, i.e., disregarding the direction of tempo change. There was a significant positive correlation between the lateral dimension and adaptation indices on $\mathrm{s} 1(r=0.934, p=0.000)$, which further substantiates the link between lesions with more left-lateralized centers of mass and the lack of initial overcorrection (Fig. 5).

The differential contribution of phase- and period correction mechanisms to the adaptation to the tempo changes was analyzed by means of two separate $2 \times 2$ ANOVAs with the between-subject factor group (patients vs. controls), and the within-subject factor tempo (faster vs. slower). For phase correction estimates, this procedure identified a main effect of group $(F(1,18)=4.617, p=0.046$, $\left.\eta_{p}{ }^{2}=0.204\right)$, indicating less efficient phase correction in patients relative to controls, independent of the direction of the tempo change, while the same type of ANOVA on period correction estimates yielded no significant results.

Correlations with lesion size and location were performed on the basis of averaged phase and period correction estimates, again disregarding the direction of tempo change. There was a significant positive correlation $(r=0.825, p=0.003)$ between the lateral dimension and period correction estimates, indicating that more
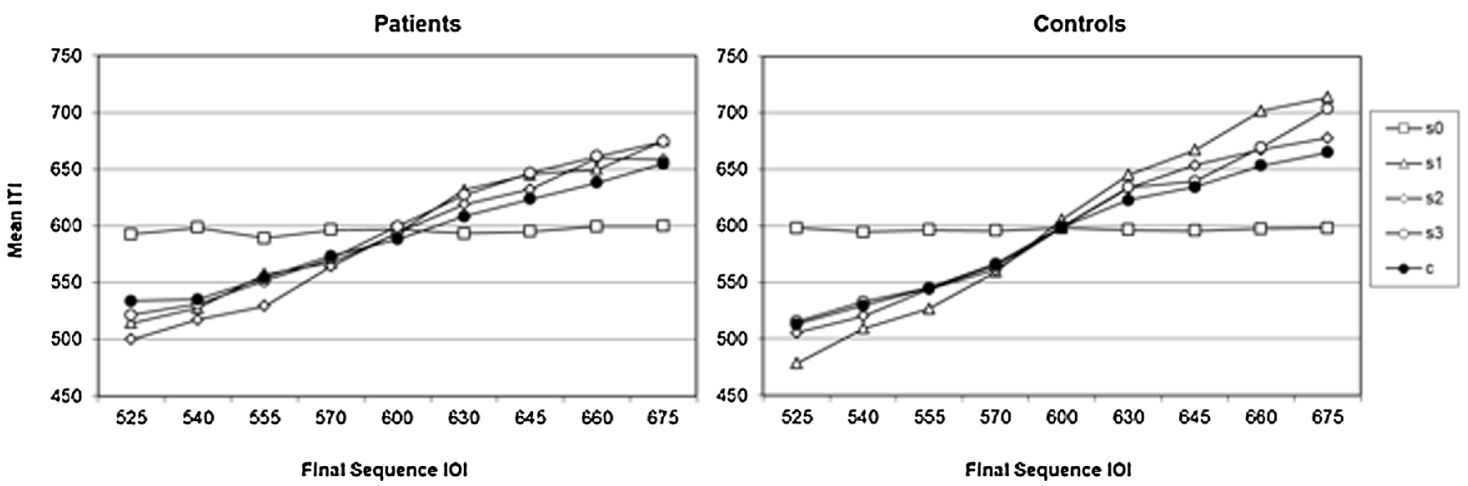

Fig. 4. Mean inter-tap-intervals (ITI) for patients and controls as a function of the final sequence inter-onset-interval (IOI). 


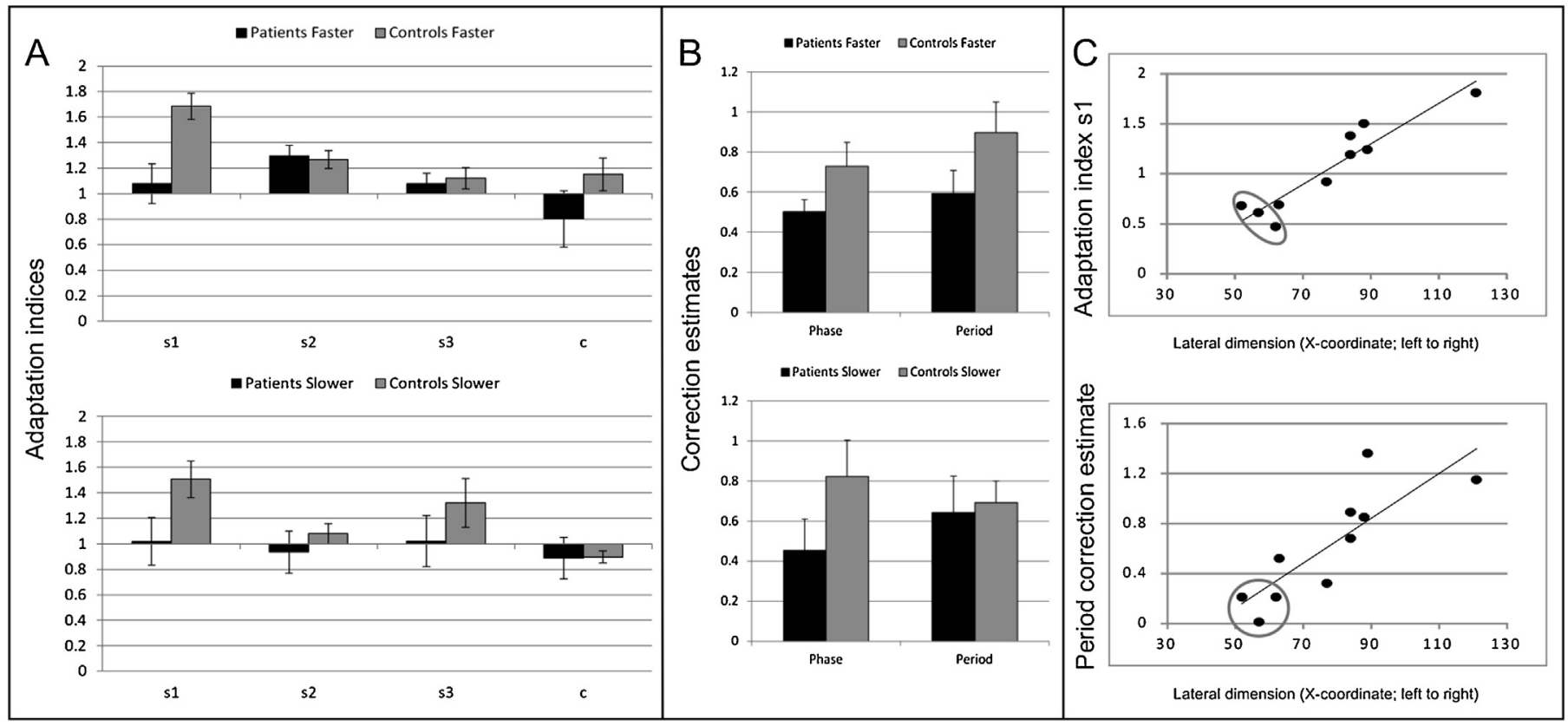

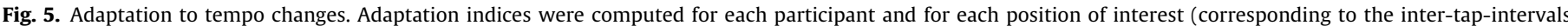

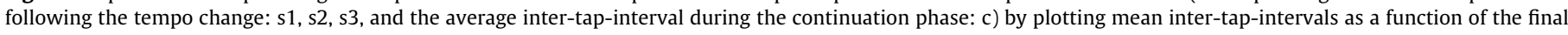

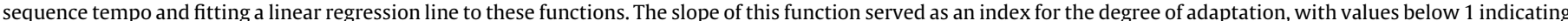

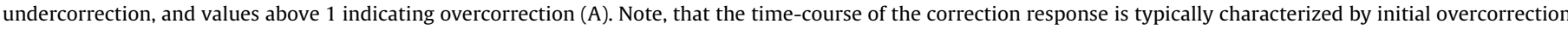

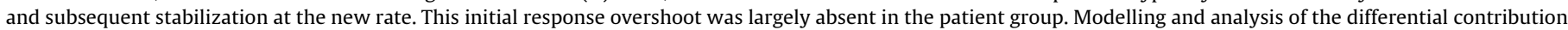

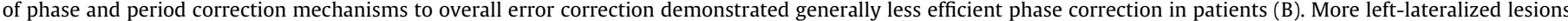

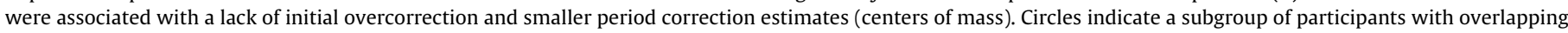
lesions in the left cerebellar crus I(C).

left-lateralized centers of mass were associated with smaller period correction estimates (Fig. 4).

\subsection{Detection of tempo changes}

To assess the ability to detect changes in the tempo of the pacing sequences, d' scores were computed for each participant (corresponding to the difference between z-transformed false alarm rates, i.e., the proportion of "no change" and "deceleration" responses for acceleration trials or "no change" and "acceleration" responses for deceleration trials, and hit rates, i.e., "acceleration" responses for tempo increases and "deceleration" responses for tempo decreases). Although d' scores for patients and controls appeared similar for tempo decreases, the performance of the two groups differed markedly for tempo increases, especially for smaller increments (Fig. 6).

A $2 \times 2 \times 4$ ANOVA with factors group, tempo, and magnitude ( \pm 30 vs. 45 vs. 60 vs. $75 \mathrm{~ms}$ ) was conducted to verify these observations. There were significant effects of group $(F(1,18)=7.753$, $\left.p=0.012, \eta_{p}{ }^{2}=0.301\right)$, tempo $\left(F(1,18)=6.655, p=0.019, \eta_{p}{ }^{2}=0.270\right)$, magnitude $\left(F(3,54)=69.606, p=0.000, \eta_{p}^{2}=0.795\right)$, and a significant interaction of these factors $(F(3,54)=7.589, p=0.001$, $\left.\eta_{p}{ }^{2}=0.297\right)$. Subsequent step-down analyses revealed an interaction of group $\times$ magnitude $\left(F(3,54)=8.730, p=0.001, \eta_{p}{ }^{2}=0.327\right)$ for faster tempi only. Direct comparisons yielded group differences for the $-30 \mathrm{~ms}(t(18)=-4.140, p=0.001, d=1.852)$ and $-45 \mathrm{~ms}(t(18)=-4.076, p=0.001, d=1.824)$ step changes and a non-significant trend for the $-60 \mathrm{~ms}$ step change $(t(18)=-1.930$, $p=0.070, d=0.864)$. The time-range ( -30 to about $-60 \mathrm{~ms})$, in which the perceptual dysfunction was present, appeared similar to the temporal shift in patients' negative mean asynchronies observed for the control sequences. Post-hoc correlation analyses between adaptation indices for s1 and d' for acceleration step changes yielded a significant correlation for $-45 \mathrm{~ms}(r=0.751$,

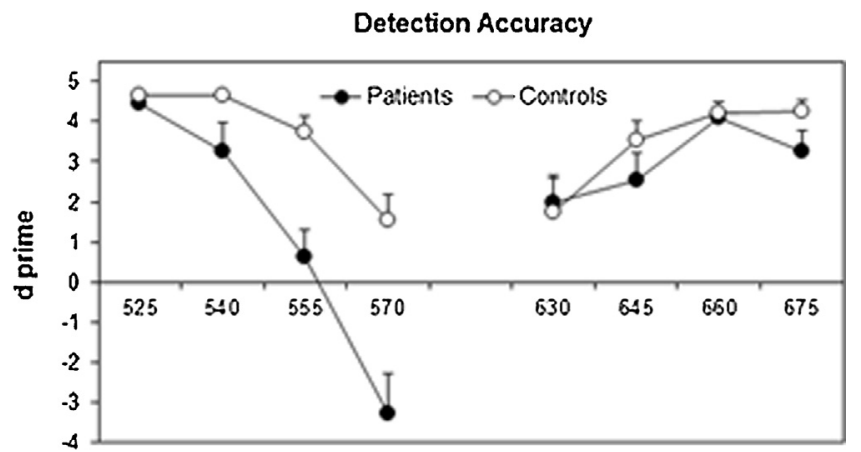

Final SOA

Fig. 6. Perceptual judgement. The ability to detect a tempo change in the pacing sequence was assessed on the basis of d' scores computed for each participant and each condition. In comparison to controls, patients showed marked differences in the detection of tempo accelerations. Statistical analyses confirmed a selective dysfunction to detect the $-30 \mathrm{~ms}$ ( $570 \mathrm{~ms}$ final SOA) and $-45 \mathrm{~ms}$ ( $555 \mathrm{~ms}$ final SOA) step changes and a trend in a similar direction for $-60 \mathrm{~ms}$.

$p=0.012$ ), indicating that smaller adaptation indices are associated with reduced perceptual sensitivity. A similar outcome for $-60 \mathrm{~ms}$ $(r=0.689, p=0.027)$ was rejected on the basis of Holm-Bonferroni adjustment. Detection accuracy for faster tempi was negatively correlated with negative mean asynchronies in patients, i.e., the larger the asynchronies, the smaller the detection accuracy for accelerations $(r=-0.725, p=0.018)$. However, this was also the case in the control group $(r=-0.662, p=0.037)$, suggesting a more fundamental relation between these measures, while the same correlations conducted for the relation between asynchronies and detection accuracy for decelerations did not yield any significant result.

In line with these findings and the results of the preceding ANOVA, the correlation analyses with lesion size and location were 
restricted to d' for faster tempi. Some potential indications of lower detection accuracy with more left-lateralized centers of mass for $-45 \mathrm{~ms}(r=0.640, p=0.046)$ and $-60 \mathrm{~ms}(r=0.707, p=0.022)$ step changes were found but rejected following Holm-Bonferroni adjustment.

\section{Discussion}

Cerebellar lesions can impair sensorimotor temporal processing although the manifestation of this overall effect depends on anatomical features (extent and location of damage), on (temporal) task characteristics and temporal processing requirements (e.g., implicit, automatic, event-based as opposed to attentiondependent, continuous, interval-based), as well as on the methods employed in the data analyses [13,33-37]. Research on this topic has hence to consider under what specific circumstances a temporal processing dysfunction emerges. However, notwithstanding functional specialization within specific cerebellar subregions, the cerebellum may instantiate a structure, which modulates activity in cerebral cortex to optimize sensory and sensorimotor behavior in terms of temporal precision and coherence, synchronization, and temporal prediction as facets of ongoing interactions with a dynamic environment.

On this account, damage to the event-based cerebellar timing system was expected to reduce the efficiency of these operations, leading to suboptimal temporal precision, which may cascade into a broad range of motor and non-motor behaviors. The current study employed self-paced spontaneous and paced finger-tapping paradigms to investigate the impact of cerebellar lesions on behavioral performance. We used a setup that places relatively high demands on sensory and sensorimotor temporal precision [24] and fitted data to a closed-loop timing model [29] that extends classical open-loop models [38] by implementing error correction mechanisms that control the temporal relationship between an internal timekeeper and external pacing events. The adaptive synchronization task in the current study employed abrupt tempo changes as opposed to gradual changes and continuously applied error correction over longer timespans, which is relatively well preserved in cerebellar and basal ganglia patients [37]. In line with the basic assumption that damage to the cerebellum modulates performance by decreasing temporal precision and leads to suboptimal behavior, we observed a number of characteristic differences between the patient and the control groups. Although some of these differences could be interpreted as instances of impaired motor function, this is not the case for all observations, especially for those that relate to reduced perceptual accuracy. Furthermore, the profile obtained for cerebellar patients differs substantially from the temporal processing dysfunction observed in basal ganglia patients tested in the same experimental setup [25].

SMT results indicate more variable self-paced generation of temporally regular events but similar tapping rates for patients as compared to healthy controls. This finding is further substantiated by more variable tapping in patients during the continuation phase of the isochronous control trials, for which the target tempo was set during the synchronization phase, but which required the self-paced generation of regular events during the continuation phase. Both forms of variability co-vary with overall lesion size. Specifically, more extensive structural damage is associated with increased variability. However, in contrast to observations in basal ganglia patients, cerebellar patients did not show indications for decreased tapping variability for SMT2 relative to SMT1, which could be interpreted as a stabilizing influence of task repetition or of the global temporal context of the intermediate sensorimotor task on variability measures [25]. This seems to suggest that the problem and the mechanism underlying increased variability in cerebellar patients is of a more persistent nature and potentially reflects damage to an automatic temporal processing system [36].

Cerebellar patients produced not only larger but also more variable negative mean asynchronies in the control sequences compared to healthy participants. The negative asynchronies allow participants to gain the subjective impression of tapping in synchrony with the pacing and therefore people are usually not aware of it $[39,40]$. The substantially larger negative mean asynchronies produced by the patients may indicate a pathological widening of the time-window in which temporal integration of the tap and the external pacing event occurs. Patients' subjective impression of being in synchrony may be the same as in controls but this effect seems to objectively reduce temporal precision in the tensof-milliseconds range preceding a critical event and it leads to inefficient task performance in the current setting.

Although the (neuro-)functional mechanisms underlying the negative mean asynchrony are still unclear [32], it has been linked to the synchronization of internal action simulation with a pacing signal $[39,40]$. Considering its role in forward-modelling and temporal processing, the cerebellum seems a likely candidate for a neural implementation of this mechanism. The current results may indicate dysfunctional matching between forward modelling and sensory input following cerebellar lesions. In this context, cerebellar "filtering" of salient events (e.g., onsets, offsets or increases in the energy-level of a stimulus) occurring in close temporal proximity (up to about $30 \mathrm{~ms}$ ) into a single temporal marker may be important to obtain distinct temporal event markers [20]. This mechanism also offers an explanation for the finding that the negative mean asynchrony is sensitive to stimulus rise-time and that people seem to use the so-called perceptual center (p-center) rather than the physical onset as a reference point for synchronization [41-43]. For this specific aspect, the current findings relate to more recent proposals concerning the functional relevance of rise-time sensitivity and the p-center in complex sensorimotor behaviors such as speech processing $[44,45]$.

Transmitted from the cerebellum via the thalamus to targets in the cerebral cortex, successive temporal markers may instantiate the precise temporal signal, which guides sensory prediction and the temporal orienting of attention $[17,20]$. In other words, these successive temporal markers may drive oscillatory activity in the sense of dynamic attending theory and/or in cortico-basal ganglia circuits implicated in interval timing and working memory $[7,18,46]$. Furthermore, one may speculate that interaction of the cerebellar mechanism with thalamic and cortical oscillatory activity imposes a fundamental constraint to the acquisition of sensory data, which is treated as "co-temporal" if it occurs within $30 \mathrm{~ms}$ [47].

In the patient and the control group, negative mean asynchronies predicted detection accuracy for tempo accelerations. The proposed role of successive cerebellar temporal event markers in the generation of sensory predictions may explain the highly asymmetrical pattern for detection accuracy in the patient group, i.e., why problems surfaced in response to events occurring during a period of anticipation as opposed to events occurring during a period of delay.

Impaired phase correction and a lack of initial overcorrection were observed for both tempo accelerations and decelerations. Phase correction is associated with registering violations of temporal expectations [32] and according to the two-way error-corrective mechanism, it is directly implemented by a motor control unit [29]. Although the position of a tempo change within a trial was in principle predictable, such knowledge seems not have been sufficient to compensate for the dysfunction observed in the patients. Cerebellar connections to early stages of sensory processing may provide the necessary temporal precision and allow for rapid (direct or automatic) implementation of phase correction [20,48]. Phase 
correction only depends on the intention to synchronize with the pacing sequence, while period correction depends also on attention and awareness of the tempo change [24]. In line with this dissociation, subliminal error correction in sensorimotor synchronization has been found to engage the cerebellar dentate nucleus, whereas supraliminal error correction recruits additional cerebellar areas as well as right inferior parietal and frontal areas [49]. Whereas basal ganglia patients had shown selectively impaired period correction [25], this type of attention-dependent error correction was spared in cerebellar patients. Period correction may hence compensate for erratic performance once dysfunctional phase correction leads to an accumulation of response discrepancies beyond a certain threshold corresponding to multiples of the actual step change over two or more subsequent ITIs (i.e., once the error magnitude becomes supraliminal). This threshold may be reflected in limits in detection accuracy (from impaired performance at -30 and $-45 \mathrm{~ms}$, and a similar trend at $-60 \mathrm{~ms}$, to preserved function at $-75 \mathrm{~ms}$ ). Compensatory engagement of attention-dependent period correction may potentially stabilize performance in patients to the extent that it becomes comparable to that of healthy controls over the course of the following taps but it seems that it cannot compensate for the dysfunctional initial (subliminal) phase correction response. Correlation analyses performed at the group level and for a subset of the patients with particularly small adaptation indices suggested a critical role of the left cerebellar crus I in the divergent pattern of results for the initial correction response. Although the functional interpretation of these results remains speculative due to the small number of participants, it is noteworthy that damage to this area in the left cerebellum had the most deteriorating effect despite the fact that all participants chose to perform the task with their right hand. This area has previously been linked to basic auditory processing [50] and resting-state functional connectivity imaging suggests that it is part of a "cognitive" network comprising the ventral cerebellar dentate and prefrontal areas [51]. Convergence of sensory and cognitive aspects in this area may suggest that these patients have the most pronounced problems with converting the auditory pacing signal into a precise temporal signal transmitted to frontal areas, including supplementary motor cortices in order to guide the stimulus-driven allocation of attention in time [52].

\section{Conclusions}

Taken together, the findings of the current study confirm a temporal processing dysfunction in patients with cerebellar lesions. The results are in line with the proposed role of the cerebellum in precise automatic, event-based temporal processing and establish a link between previous work in cerebellar patients and a sensory predictive account of cerebellar function. The specific pattern of results seems compatible with the proposal of a general cerebellar function in optimizing the temporal component of ongoing interactions with a dynamic environment.

\section{Acknowledgements}

The authors would like to thank Anne-Kathrin Franz and Heike Boethel for support during data acquisition, and Anika Stockert for support in the preparation of the clinical data.

\section{References}

[1] C.H. Chen, R. Fremont, E.E. Arteaga-Bracho, K. Khodakhah, Short latency cerebellar modulation of the basal ganglia, Nat. Neurosci. 17 (2014) 1767-1777

[2] R.P. Dum, P.L. Strick, An unfolded map of the cerebellar dentate nucleus and its projections to the cerebral cortex, J. Neurophysiol. 89 (2003) 634-639.

[3] E. Hoshi, L. Tremblay, J. Feger, P.L. Carras, P.L. Strick, The cerebellum communicates with the basal ganglia, Nat. Neurosci. 8 (2005) 1491-1493.
[4] F.A. Middleton, P.L. Strick, Basal ganglia and cerebellar loops: motor and cognitive circuits, Brain Res. Rev. 31 (2000) 236-250.

[5] R.D. Proville, M. Spolidoro, N. Guyon, G.P. Dugue, F. Selimi, P. Isope, D. Popa, C. Lena, Cerebellum involvement in cortical sensorimotor circuits for the control of voluntary movements, Nat. Neurosci. 17 (2014) 1233-1241.

[6] P.L. Strick, R.P. Dum, J.A. Fiez, Cerebellum and nonmotor function, Annu. Rev. Neurosci. 32 (2009) 413-432.

[7] C.V. Buhusi, W.H. Meck, What makes us tick? Functional and neural mechanisms of interval timing, Nat. Rev. Neurosci. 6 (2005) 755-785.

[8] J.T. Coull, R. Cheng, W.H. Meck, Neuroanatomical and neurochemical substrates of timing, Neuropsychopharmacology 36 (2011) 3-25.

[9] R.B. Ivry, J.E. Schlerf, Dedicated and intrinsic models of time perception, Trends Cogn. Sci. 12 (2008) 273-280.

[10] H. Merchant, D.L. Harrington, W.H. Meck, Neural basis of the perception and estimation of time, Annu. Rev. Neurosci. 36 (2013) 313-336.

[11] M. Wiener, P. Turkeltaub, H.B. Coslett, The image of time: a voxel-wise meta-analysis, Neuroimage 49 (2010) 1728-1740.

[12] R.B. Ivry, The representation of temporal information and motor control, Curr. Opin. Neurobiol. 6 (1996) 851-857.

[13] R.M.C. Spencer, H.N. Zelaznik, J. Diedrichsen, R.B. Ivry, Disrupted timing of discontinuous but not continuous movements by cerebellar lesions, Science 300 (2003) 1437-1439.

[14] R.M.C. Spencer, R.B. Ivry, Cerebellum and timing, in: M. Manto, D.L. Gruol, J.D. Schmahmann, N. Koibuchi, F. Rossi (Eds.), Handbook of the Cerebellum and Cerebellar Disorders, Springer, Dordrecht, 2013, pp. 1201-1219.

[15] E. Courchesne, G. Allen, Prediction and preparation, fundamental functions of the cerebellum, Learn. Mem. 4 (1997) 1-35.

[16] D.M. Wolpert, R.C. Miall, M. Kawato, Internal models in the cerebellum, Trends Cogn. Sci. 2 (1998) 338-347.

[17] J.X. O'Reilly, M.M. Mesulam, A.C. Nobre, The cerebellum predicts the timing of perceptual events, J. Neurosci. 28 (2008) 2252-2260

[18] E. Large, M.R. Jones, The dynamics of attending: how people track time-varying events, Psychol. Rev. 106 (1999) 119-159.

[19] M. Manto, J.M. Bower, A.B. Conforto, J.M. Delgado-García, S.N. da Guarda, M Gerwig, C. Habas, N. Hagura, R.B. Ivry, P. Mariën, M. Molinari, E. Naito, D.A. Nowak, N. Oulad Ben Taib, D. Pelisson, C.D. Tesche, C. Tilikete, D. Timmann, Consensus paper: roles of the cerebellum in motor control-the diversity of ideas on cerebellar involvement in movement, Cerebellum 11 (2012) 457-487.

[20] M. Schwartze, S.A. Kotz, A dual-pathway neural architecture for specific temporal prediction, Neurosci. Biobehav. Rev. 37 (2013) 2587-2596.

[21] J.M. Bower, The cerebellum as sensory acquisition controller, Hum. Brain Map. 2 (1995) 255-256.

[22] J. Gao, L.M. Parsons, J.M. Bower, J. Xiong, P.T. Fox, Cerebellum implicated in sensory acquisition and discrimination rather than motor control, Science 272 (1996) 545-547.

[23] V.B. Penhune, R.J. Zatorre, A.C. Evans, Cerebellar contributions to motor timing: a PET study of auditory and visual rhythm reproduction, J. Cogn. Neurosci. 10 (1998) 752-765.

[24] B.H. Repp, P.E. Keller, Adaptation to tempo changes in sensorimotor synchronization: effects of intention, attention, and awareness, Q. J. Exp. Psychol. 57A (2004) 499-521.

[25] M. Schwartze, P.E. Keller, A.D. Patel, S.A. Kotz, The impact of basal ganglia lesions on sensorimotor synchronization spontaneous motor tempo, and the detection of tempo changes, Behav. Brain Res. 216 (2011) 685-691.

[26] J.P. Mugler 3rd, J.R. Brookeman, Three-dimensional magnetization-prepared rapid gradient-echo imaging (3D MP RAGE), Magn. Reson. Med. 15 (1990) 152-157.

[27] K.J. Friston, J. Ashburner, C.D. Frith, J. Poline, J.D. Heather, R.S.J. Frackowiak, Spatial registration and normalization of images, Hum. Brain Map. 2 (1995) $165-189$.

[28] C. Rorden, M. Brett, Stereotaxic display of brain lesions, Behav. Neurol. 12 (2000) 191-200.

[29] J. Mates, A model of synchronization of motor acts to a stimulus sequence I. Timing and error corrections, Biol. Cybern. 70 (1994) 463-467.

[30] S. Holm, A simple sequentially rejective multiple test procedure, Scand. J. Stat. 6 (1979) 65-70.

[31] B.H. Repp, Tapping in synchrony with a perturbed metronome: the phase correction response to small and large phase shifts as a function of tempo, J. Motor Behav. 43 (2011) 213-227.

[32] B.H. Repp, Y. Su, Sensorimotor synchronization: a review of recent research (2006-2012), Psychon. Bull. Rev. 20 (2013) 403-452.

[33] D.L. Harrington, R.R. Lee, L.A. Boyd, S.Z. Rapcsak, R.T. Knight, Does the representation of time depend on the cerebellum? Effect of cerebellar stroke, Brain 127 (2004) 561-574.

[34] R.B. Ivry, R.M.C. Spencer, H.N. Zelaznik, J. Diedrichsen, The cerebellum and event timing, Ann. N. Y. Acad. Sci. 978 (2002) 302-317.

[35] R.B. Ivry, R.M.C. Spencer, Evaluating the role of the cerebellum in temporal processing: beware of the null hypothesis, Brain 127 (2004) e13.

[36] P.A. Lewis, C.R. Miall, Distinct systems for automatic and cognitively controlled time measurement: evidence from neuroimaging, Curr. Opin. Neurobiol. 13 (2003) 250-255.

[37] M.C. van der Steen, M. Schwartze, S.A. Kotz, P.E. Keller, Modeling effects of cerebellar and basal ganglia lesions on adaptation and anticipation during sensorimotor synchronization, Ann. N. Y. Acad. Sci. 1337 (2015) 101-110. 
[38] A.M. Wing A.B. Kristofferson, Response delays and the timing of discrete motor responses, Percept. Psychophys. 14 (1973) 5-12.

[39] G. Aschersleben, Temporal control of movements in sensorimotor synchronization, Brain Cogn. 48 (2000) 66-79.

[40] R.H. Repp, Sensorimotor synchronization: a review of the tapping literature, Psychon. Bull. Rev. 12 (2005) 969-992.

[41] S.M. Marcus, Acoustic determinants of perceptual center (P-center) location, Percept. Psychophys. 30 (1981) 247-256.

[42] J. Morton, S.M. Marcus, C.R. Frankish, Perceptual centers (P-centers), Psychol. Rev. 83 (1976) 405-408.

[43] P.G. Vos, J. Mates, The perceptual centre of a stimulus as the cue for synchronization to a metronome: evidence from asynchronies, Q. J. Exp. Psychol. 48A (1995) 1024-1040.

[44] U. Goswami, A temporal sampling framework for developmental dyslexia, Trends Cogn. Sci. 15 (2011) 3-10.

[45] S.A. Kotz, M. Schwartze, Cortical speech processing unplugged: a timely subcortico-cortical framework, Trends Cogn. Sci. 14 (2010) 392-399.

[46] B. Gu, H. van Rijn, W.H. Meck, Oscillatory multiplexing of neural population codes for interval timing and working memory, Neurosci. Biobehav. Rev. 48 (2015) 160-185.
[47] E. Pöppel, A hierarchical model of temporal perception, Trends Cogn. Sci. 1 (1997) 56-61.

[48] M.J. Hove, R. Balasubramaniam, P.E. Keller, The time course of phase correction: a kinemtatic investigation of motor adjustment to timing perturbations during sensorimotor synchronization, J. Exp. Psychol. Hum. Percept. Perform. 40 (2014) 2243-2251.

[49] J.D. Bijsterbosch, K. Lee, M.D. Hunter, D.T. Tsoi, S. Lankappa, I.D. Wilkinson, A.T. Barker, P.W.R. Woodruff, The role of the cerebellum in sub- and supraliminal error correction during sensorimotor synchronization: evidence from fMRI and TMS, J. Cogn. Neurosci. 23 (2011) 1100-1112.

[50] A. Petacchi, A.R. Laird, P.T. Fox, J.M. Bower, Cerebellum and auditory function: an ALE meta-analysis of functional neuroimaging studies, Hum. Brain Map. 25 (2005) 118-128.

[51] J.A. Bernard, S.J. Peltier, B.L. Benson, J.L. Wiggins, S.M. Jaeggi, M. Buschkuehl, J Jonides, C.S. Monk, R.D. Seidler, Disscociable functional networks of the human dentate nucleus, Cereb. Cortex 24 (2014) 2151-2159.

[52] M. Schwartze, K. Rothermich, S.A. Kotz, Functional dissociation of pre-SMA and SMA-proper in temporal processing, Neuroimage 60 (2012) 290-298. 